\title{
The Effect of an Open Carpal Tunnel Release on Thumb CMC Biomechanics
}

\author{
Marc A. Tanner, Bryan P. Conrad, Paul C. Dell, and Thomas W. Wright
}

Department of Orthopaedics and Rehabilitation, Orthopaedics and Sports Medicine Institute, University of Florida, Gainesville, FL 32608, USA

Correspondence should be addressed to Thomas W. Wright, wrightw@ortho.ufl.edu

Received 19 April 2012; Revised 23 October 2012; Accepted 24 October 2012

Academic Editor: Allen L. Carl

Copyright ( 2012 Marc A. Tanner et al. This is an open access article distributed under the Creative Commons Attribution License, which permits unrestricted use, distribution, and reproduction in any medium, provided the original work is properly cited.

Purpose. We have observed worsening thumb pain following carpal tunnel release (CTR) in some patients. Our purpose was to determine the effect of open CTR on thumb carpometacarpal (CMC) biomechanics. Methods. Five fresh-frozen cadaver arms with intact soft tissues were used. Each specimen was secured to a jig which fixed the forearm at $45^{\circ}$ supination, and the wrist at $20^{\circ}$ dorsiflexion, with thumb pointing up. The thumb was axially loaded with a force of $130 \mathrm{~N}$. We measured 3D translation and rotation of the trapezium, radius, and first metacarpal, before and after open CTR. Motion between radius and first metacarpal, radius and trapezium, and first metacarpal and trapezium during loading was calculated using rigid body mechanics. Overall stiffness of each specimen was determined. Results. Total construct stiffness following CTR was reduced in all specimens but not significantly. No significant changes were found in adduction, pronation, or dorsiflexion of the trapezium with respect to radius after open CTR. Motion between radius and first metacarpal, between radius and trapezium, or between first metacarpal and trapezium after open CTR was not decreased significantly. Conclusion. From this data, we cannot determine if releasing the transverse carpal ligament alters kinematics of the $\mathrm{CMC}$ joint.

\section{Introduction}

Carpal tunnel syndrome and basal joint arthritis often coexist [1]. A study of 246 patients with basal joint arthritis of the thumb reported that $39 \%$ of the study patients also had carpal tunnel syndrome [2]. Anecdotally, we have observed worsening thumb carpometacarpal (CMC) pain in some patients with previously asymptomatic (or minimally symptomatic) first $\mathrm{CMC}$ arthritis after undergoing a carpal tunnel release. The thumb CMC joint is the most common site for reconstruction in the upper extremity secondary to osteoarthritis [3]. It is a semiconstrained joint composed of two saddle-shaped articulations with opposing axes perpendicular to each other. Minimal congruence and bony stability allow for a wide range of motion [4].

The anterior border of the carpal tunnel is formed by the transverses carpal ligament (TCL) [5]. The transverse carpal ligament proper inserts into the scaphoid tubercle and trapezial ridge radially and the hamulus and pisiform ulnarly [6]. Studies have noted increase in carpal tunnel volume, increase in the carpal arch width, and an overall decrease in carpal stiffness after carpal tunnel release (CTR) [6-12]. Rotational changes of the hamate, pisiform, and the trapezium have also been reported, as well as an increase in the distance between the trapezium and the hook of the hamate [5]. Previous research suggests a direct relationship between widening of the transverse carpal arch and loss of grip strength [13]. Pillar pain is a common complaint after CTR [14], perhaps as a result of the division of the TCL during surgery [6].

Pain originates most commonly over the pisotriquetral joint, possibly secondary to displacement of the pisiform or alteration of forces over the joint $[11,15]$. The same type of biomechanical changes could occur with the trapezium and the CMC joint. The purpose of this study is to determine the effect of open CTR on the kinematics of the trapezium 
and first CMC joint. Our hypothesis was that a CTR will allow rotation of the trapezium altering the biomechanics of the first CMC joint. These changes could result in the clinical observation of increased first CMC joint pain in patients with previous subclinical CMC arthritis.

\section{Materials and Methods}

Five fresh-frozen cadaver arms with intact soft tissues were used. There were three male specimens and two female specimens (donor age was not available). Each specimen was secured to a custom-made jig with 3.0 threaded Steinman pins, two at the distal radius and two placed in the second metacarpal (Figure 1).

The jig fixed the forearm at $45^{\circ}$ supination and was designed to allow free motion at the thumb CMC joint and radiocarpal joint while the wrist was fixed in $20^{\circ}$ of dorsiflexion. A threaded $2.0 \mathrm{~mm} \mathrm{~K}$-wire was placed from the tip of the thumb distal phalanx into the thumb metacarpal, leaving $2 \mathrm{~cm}$ of wire out of the skin. The wire out of the skin was placed into an MTS machine (MTS Systems, Eden Prairie, $\mathrm{MN}$ ) and the jig secured to the base of the testing machine.

An electromagnetic tracking system (Liberty, Polhemus Inc, Colchester, VT) was used to measure the 3D translation and rotation of the trapezium, radius, and first metacarpal. Electromagnetic sensors were attached to the radius and first metacarpal using custom-made fiberglass brackets. A threaded K-wire was placed into the trapezium, ensuring that the joint capsule remained intact, and a third electromagnetic sensor was attached to the K-wire to track the trapezium. The electromagnetic source was positioned within $20 \mathrm{~cm}$ of the sensors to minimize the effect of distortion created by the testing machine and jig. Internal electronics of the Polhemus Liberty system are capable of detecting distortions and, when present, the testing setup was adjusted to eliminate them. After loading at $10 \mathrm{~N}$ once to remove any slack, the thumb was axially loaded with a $130 \mathrm{~N}$ force.

The total motion of the first metacarpal relative to the radius, between the radius and trapezium, and between the first metacarpal and the trapezium during loading was calculated using rigid body mechanics. The stiffness of each specimen was also determined by measuring the slope of the load-displacement curve during loading. The measurements were collected from each specimen before and after an open CTR. An open CTR was performed in the standard fashion and verified visually and with palpation. The release was performed without removing the specimen from the jig. A paired $t$-test was used to determine differences in rotation and translation of the specimen befor and after CTR.

\section{Result}

Motion and stiffness data is presented in Table 1.

All five specimens demonstrated a reduction in the total construct stiffness following CTR; however, the difference was not statistically significant $(P=0.1)$. The average adduction, pronation, and dorsiflexion of the trapezium with

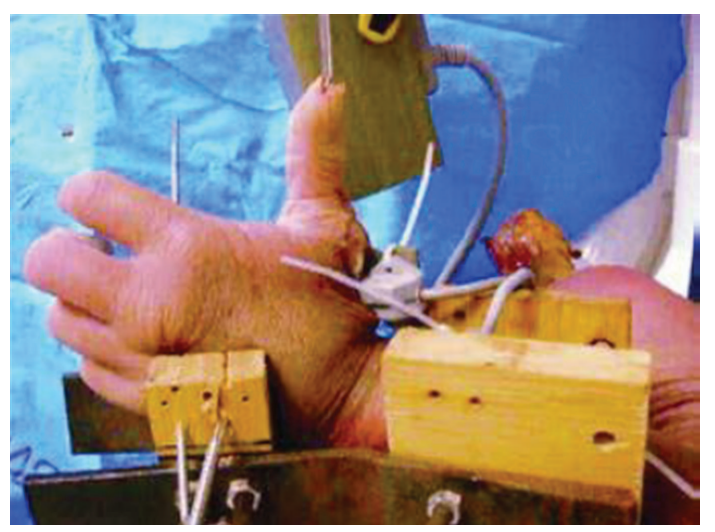

FIGURE 1: Photograph of a specimen secured in the custom-made jig. The forearm is at 45 -degree supination and the wrist at 20degree dorsiflexion, thumb pointing up.

respect to the radius did not change significantly after open CRT. No significant decrease in range of motion was measured between the radius and first metacarpal $(P=0.12)$, between the radius and trapezium $(P=0.32)$, or between the first metacarpal and trapezium $(P=0.55)$ after an open CTR.

\section{Discussion}

The purpose of this study was to determine the effect of open CTR on the kinematics of the first CMC joint. Our hypothesis that a CTR would cause rotation of the trapezium altering the biomechanics of the first CMC joint was not supported by the data from this study. Less stiffness was seen in all of the specimens at the radius-metacarpal interface after CTR; however, the difference was not statistically significant. The magnitude of change at the trapezium that would cause symptoms is not known. It may have been that the small changes in stiffness we observed were not statistically significant because the study did not have sufficient power. We do not know what decrease in stiffness would be enough to account for a perceived increase in postoperative pain at the thumb CMC joint in patients with prior subclinical CMC arthritis. Likewise rotation of the trapezium would likely affect the scaphotrapezial trapezoid (STT) joint. This could also cause pain near the base of the thumb. We did not specifically evaluate the STT joint in this study.

The only previous study which addressed this issue was presented in 2009 at the American Academy of Orthopaedic Surgery meeting [16]. Changes in rotation of the trapezium were noted in their study but were not statistically significant. Outward rotational changes in the trapezium of 2.25 degrees $( \pm 1.6$ degrees $)$ were found after CTR. They also reported rotational changes in the pisiform and the magnitude of these changes was greater than those found at the trapezium (3.83 degrees and 4.5 degrees, resp.).

A weakness of this study is the small sample size and the inherent variability of cadaveric specimens. Even though the specimens were preconditioned, it is probable that in the normal physiologic state some biomechanical changes may 
TABLE 1: Summary of mechanical properties for each combination of bones before and after open carpal tunnel release.

\begin{tabular}{|c|c|c|c|c|}
\hline Measurement & $\begin{array}{c}\text { Intact } \\
(\text { mean } \pm S D)\end{array}$ & $\begin{array}{l}\text { After release } \\
(\text { mean } \pm S D)\end{array}$ & Difference & $P$ value \\
\hline Rad-Met1 $R_{x}$ (adduction) (deg) & $2.2 \pm 1.3$ & $2.1 \pm 1.6$ & $6.6 \%$ & 0.69 \\
\hline Rad-Met1 $R_{y}$ (pronation) (deg) & $0.7 \pm 0.6$ & $0.7 \pm 0.4$ & $-7.9 \%$ & 0.56 \\
\hline Rad-Met1 $R_{z}$ (dorsiflexion) (deg) & $3.5 \pm 1.4$ & $3.4 \pm 1.5$ & $3.0 \%$ & 0.78 \\
\hline Rad-Met1 $X$ (dorsal deviation) (mm) & $1.9 \pm 1.6$ & $2 \pm 1.7$ & $-3.3 \%$ & 0.48 \\
\hline Rad-Met1 Y (subluxation) (mm) & $1.4 \pm 0.7$ & $1.5 \pm 0.7$ & $-9.1 \%$ & 0.12 \\
\hline Rad-Met1 $Z$ (ulnar deviation) (mm) & $1.5 \pm 0.7$ & $1.5 \pm 0.6$ & $-5.5 \%$ & 0.54 \\
\hline Rad-Trap $R_{x}$ (adduction) (deg) & $1.9 \pm 1.1$ & $2.1 \pm 1.2$ & $-10.2 \%$ & 0.17 \\
\hline Rad-Trap $R_{y}$ (pronation) (deg) & $2.9 \pm 2.2$ & $2.9 \pm 2.4$ & $-0.9 \%$ & 0.83 \\
\hline Rad-Trap $R_{z}$ (dorsiflexion) (deg) & $2.0 \pm 2.7$ & $1.9 \pm 2.3$ & $4.1 \%$ & 0.70 \\
\hline Rad-Trap $X$ (dorsal deviation) $(\mathrm{mm})$ & $1.3 \pm 0.9$ & $1.1 \pm 0.8$ & $11.0 \%$ & 0.13 \\
\hline Rad-Trap Y (subluxation) (mm) & $1.0 \pm 1.1$ & $1.0 \pm 1.1$ & $-6.0 \%$ & 0.32 \\
\hline Rad-Trap $Z$ (ulnar deviation) (mm) & $1.2 \pm 0.5$ & $1.3 \pm 0.6$ & $-8.6 \%$ & 0.31 \\
\hline Trap-Met1 $R_{x}$ (adduction) (deg) & $9.0 \pm 9.8$ & $8.6 \pm 8.8$ & $5.1 \%$ & 0.60 \\
\hline Trap-Met1 $R_{y}$ (pronation) (deg) & $2.7 \pm 1.7$ & $2.6 \pm 2.0$ & $2.5 \%$ & 0.83 \\
\hline Trap-Met1 $R_{z}$ (dorsiflexion) (deg) & $12 \pm 11.2$ & $11.9 \pm 11.2$ & $1.3 \%$ & 0.80 \\
\hline Trap-Met1 $X$ (dorsal deviation) (mm) & $0.5 \pm 0.2$ & $0.6 \pm 0.5$ & $-32.4 \%$ & 0.36 \\
\hline Trap-Met1 $Y$ (subluxation) (mm) & $1.2 \pm 1.2$ & $1.2 \pm 1.2$ & $-2.8 \%$ & 0.55 \\
\hline Trap-Met1 $Z$ (ulnar deviation) (mm) & $0.7 \pm 0.4$ & $0.6 \pm 0.4$ & $5.3 \%$ & 0.39 \\
\hline Overall specimen stiffness (N/mm) & $48.9 \pm 23.4$ & $41.4 \pm 18.4$ & $15.3 \%$ & 0.11 \\
\hline
\end{tabular}

Rad-Met: radius to 1st metacarpal; Rad-Trap: radius to trapezium; Trap-Met: trapezium to 1st metacarpal.

occur over time as the constraints further stretch out. The native musculature was not used to dynamically load the CMC. In a cadaver specimen it is challenging to reproduce physiological loading and it is possible that the tested condition does not reproduce normal CMC loading. The strengths of the study are that the surrounding soft tissues of the forearm were not disturbed and the carpal tunnel release was performed without removing the arm from the custom jig. Many other factors come into play concerning the clinical onset of 1st CMC joint pain including deconditioning or increased activity level once the patient has recovered from carpal tunnel surgery.

Our goal was to explore changes in kinematics at the trapezium following CTR. These changes could be a result of the changes in the anatomic relationship due to the release of the TCL affecting the forces at its insertion onto the trapezial ridge. Small rotational changes could affect the normal kinematics of the CMC joint during physiologic loading. Based on the methodology of this cadaver study, we were unable to prove our hypothesis that releasing the TCL would result in kinematic changes of the trapezium with secondary effects on the 1st CMC joint that could be responsible for postoperative pain in a previous arthritic but asymptomatic CMC joint.

\section{Ethical Approval}

No human subjects were included in this study; therefore, IRB approval was not required. At the time this project began, no approval was needed for cadaver study.

\section{Conflict of Interests}

There was no financial or other support received for this research, and none of the authors have a conflict of interests.

\section{References}

[1] C. P. Melone Jr., B. Beavers, and A. Isani, "The basal joint pain syndrome," Clinical Orthopaedics and Related Research, vol. 220, pp. 58-67, 1987.

[2] T. M. Florack, R. J. Miller, V. D. Pellegrini, R. I. Burton, and M. G. Dunn, "The prevalence of carpal tunnel syndrome in patients with basal joint arthritis of the thumb," Journal of Hand Surgery. Series A, vol. 17, no. 4, pp. 624-630, 1992.

[3] A. L. Armstrong, J. B. Hunter, and T. R. Davis, "The prevalence of degenerative arthritis of the base of the thumb in postmenopausal women," Journal of Hand Surgery. Series B, vol. 19, no. 3, pp. 340-341, 1994.

[4] A. M. Hollister, W. L. Buford, L. M. Myers, D. J. Giurintano, and A. Novick, "The axes of rotation of the thumb carpometacarpal joint," Journal of Orthopaedic Research, vol. 10, no. 3, pp. 454-460, 1992.

[5] J. J. Brooks, J. R. Schiller, S. D. Allen, and E. Akelman, "Biomechanical and anatomical consequences of carpal tunnel release," Clinical Biomechanics, vol. 18, no. 8, pp. 685-693, 2003.

[6] T. K. Cobb, B. K. Dalley, R. H. Posteraro, and R. C. Lewis, "Anatomy of the flexor retinaculum," Journal of Hand Surgery. Series A, vol. 18, no. 1, pp. 91-99, 1993.

[7] J. A. Richman, R. H. Gelberman, B. L. Rydevik et al., "Carpal tunnel syndrome: morphologic changes after release of the transverse carpal ligament," Journal of Hand Surgery. Series A, vol. 14 , no. 5, pp. 852-857, 1989. 
[8] G. R. Fisk, "The influence of the transverse carpal ligament (flexor retinaculum) on carpal stability," Annales de Chirurgie de la Main, vol. 3, no. 4, pp. 297-299, 1984.

[9] F. K. Fuss and T. F. Wagner, "Biomechanical alterations in the carpal arch and hand muscles after carpal tunnel release: a further approach toward understanding the function of the flexor retinaculum and the cause of postoperative grip weakness," Clinical Anatomy, vol. 9, no. 2, pp. 100-108, 1996.

[10] H. Seradge and E. Seradge, "Piso-triquetral pain syndrome after carpal tunnel release," Journal of Hand Surgery. Series A, vol. 14, no. 5, pp. 858-862, 1989.

[11] M. Garcia-Elias, K. N. An, W. P. Cooney, R. L. Linscheid, and E. Y.S. Chao, "Stability of the transverse carpal arch: an experimental study," Journal of Hand Surgery. Series A, vol. 14, no. 2, part 1, pp. 277-282, 1989.

[12] S. F. Viegas, A. Pollard, and K. Kaminksi, "Carpal arch alteration and related clinical status after endoscopic carpal tunnel release," Journal of Hand Surgery. Series A, vol. 17, no. 6, pp. 1012-1016, 1992.

[13] G. M. Gartsman, J. C. Kovach, C. C. Crouch, P. C. Noble, and J. B. Bennett, "Carpal arch alteration after carpal tunnel release," Journal of Hand Surgery, vol. 11, no. 3, pp. 372-374, 1986.

[14] K. S. Ludlow, J. L. Merla, J. A. Cox, and L. N. Hurst, "Pillar pain as a postoperative complication of carpal tunnel release: a review of the literature," Journal of Hand Therapy, vol. 10, no. 4, pp. 277-282, 1997.

[15] R. G. Eaton and J. W. Littler, "Ligament reconstruction for the painful thumb carpometacarpal joint," Journal of Bone and Joint Surgery. Series A, vol. 55, no. 8, pp. 1655-1666, 1973.

[16] J. R. Schiller, J. J. Brooks, D. C. Moore, J. J. Crisco, and E. Akelman, "Three dimensional carpal kinematics after carpal tunnel release," in Proceedings of the Annual Meeting of the American Academy of Orthopaedic Surgeons, Las Vegas, Nev, USA, February 2009. 


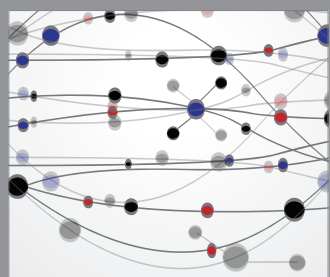

The Scientific World Journal
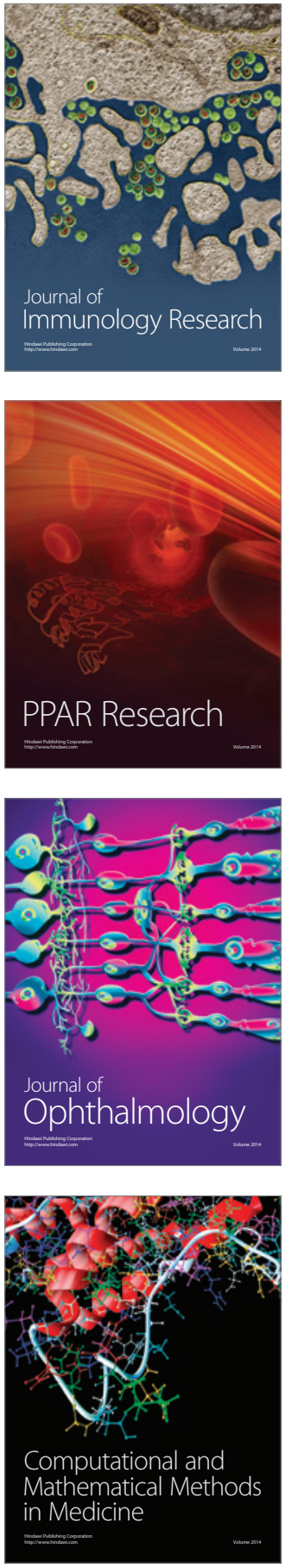

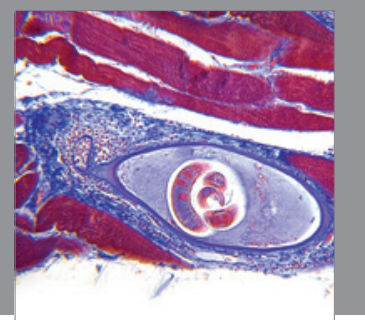

Gastroenterology

Research and Practice
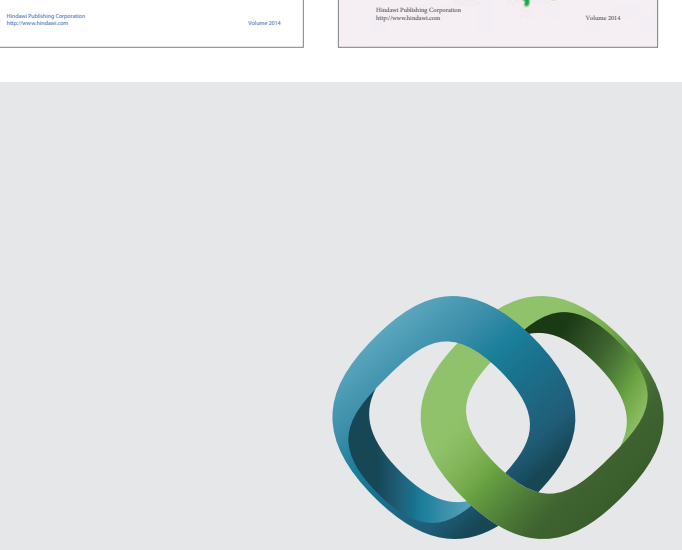

\section{Hindawi}

Submit your manuscripts at

http://www.hindawi.com
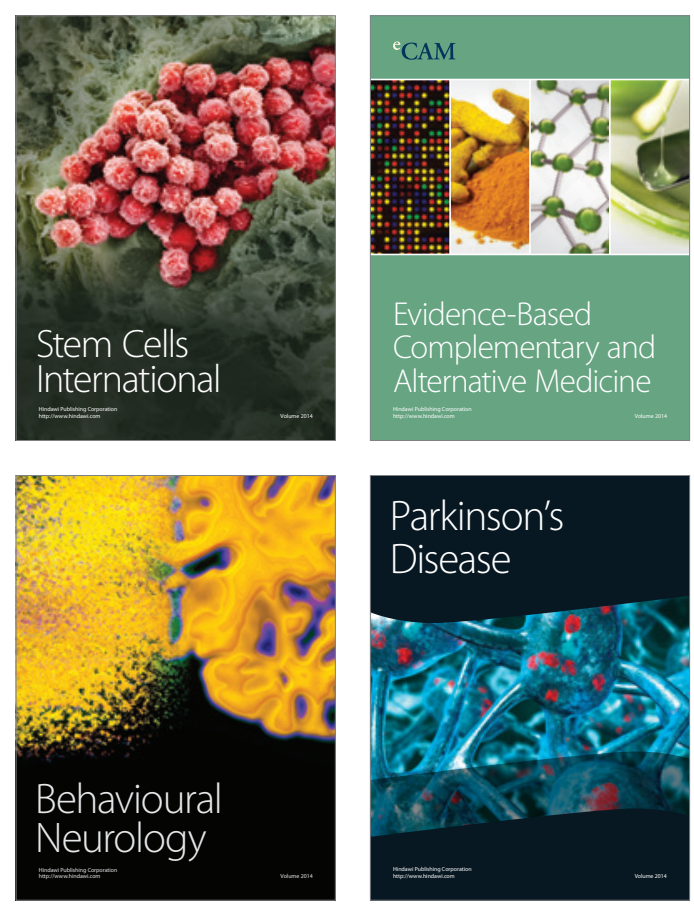

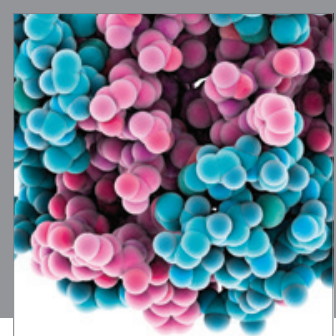

Journal of
Diabetes Research

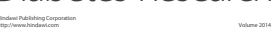

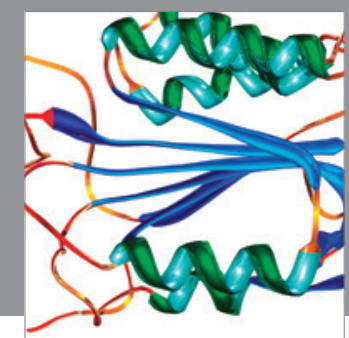

Disease Markers
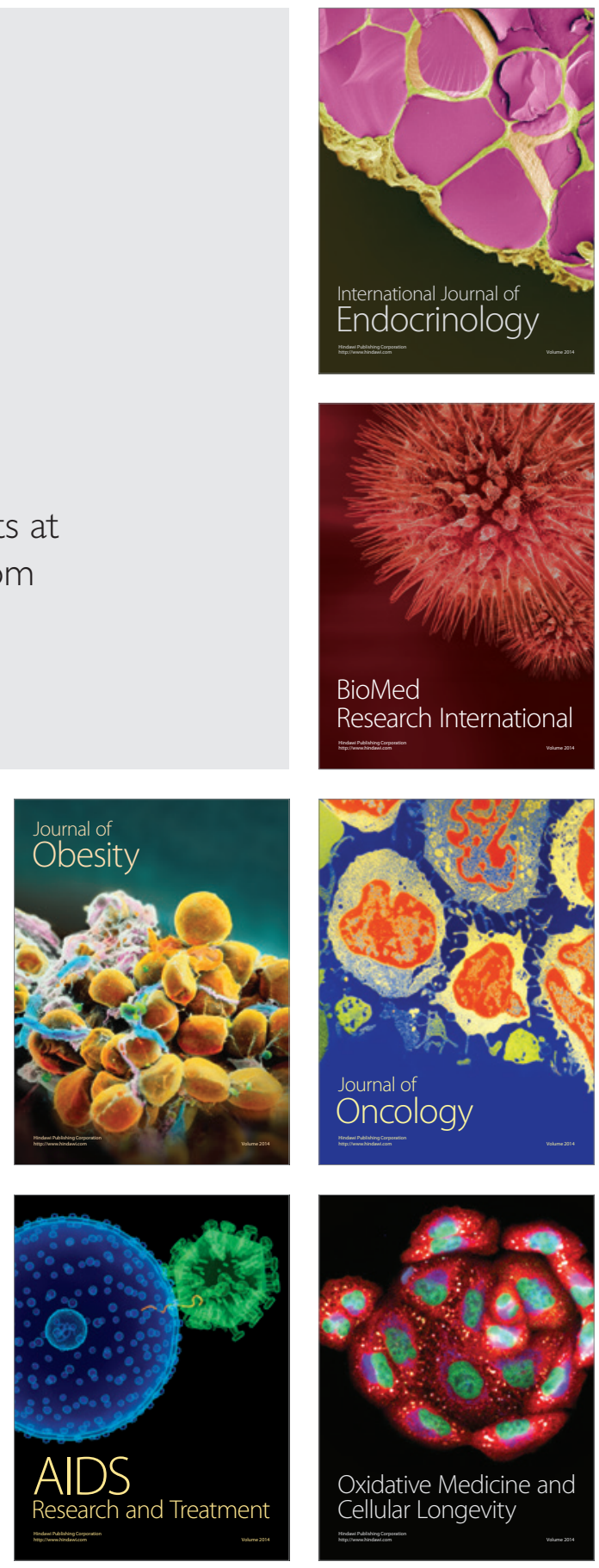